\title{
Uso da simulação computacional para ensino e aprendizagem de anatomia para aulas EAD nos cursos de Saúde
}

\author{
Use of computer simulation for teaching and learning anatomy for distance learning classes in
}

\section{Health courses}

Uso de simulación por computadora para enseñar y aprender anatomía para clases de educación a distancia en cursos de Salud

\section{Resumo}

A humanidade passa por uma movimentação tecnológica nunca vista até o momento, em que a informação e a comunicação alcançam um plano essencial na vida das pessoas. Sendo assim, destaca-se a necessidade das escolas em acompanhar a evolução tecnológica e tirar o máximo de proveito dos benefícios que esta é capaz de proporcionar. Nesse contexto, o presente trabalho tem como tema, o uso de Tecnologias da Informação e Comunicação (TIC) no ambiente de ensino-aprendizagem da disciplina de Anatomia, mais especificamente a utilização de simulação computacional nas aulas EAD nos cursos de Saúde. Foi realizado um estudo de revisão bibliográfica através da plataforma científica Scielo, utilizando-se as seguintes palavras-chave: "Simulação Computacional", "Ensino a Distância" e "Ensino de Anatomia". Verificou-se que o papel da simulação computacional no estudo do corpo humano, no qual contribui com o aumento da qualidade dos resultados obtidos e a redução de custos e dificuldades envolvidos, bem como as diversas vantagens de observação de peças com múltiplos níveis e angulação e anatomia virtual não destrutiva. Foi possível verificar que no que se refere o processo de aprendizagem do discente, a visualização das peças anatômicas em mais de uma dimensão auxilia a materializar conceitos abstratos, ampliando as possibilidades de compreensão de conceitos complexos de Anatomia.

Palavras-chave: Ensino de anatomia; Ferramentas pedagógicas; Educação a distância. 


\begin{abstract}
Humanity is going through a technological movement never seen before, in which information and communication reach an essential level in people's lives. Thus, there is a need for schools to keep up with the technological evolution and make the most of the benefits that it can provide. In this context, the present work has as its theme the use of Information and Communication Technologies (ICT') in the teaching-learning environment of the Anatomy discipline, more specifically the use of computer simulation in distance education classes in Health courses. A literature review was carried out using the scientific platform Scielo, using the following keywords: "Computer Simulation", "Distance Learning" and "Anatomy Teaching". It was verified that the role of computer simulation in the study of the human body, in which it contributes with the increase of quality of the results obtained and the reduction of costs and difficulties involved, as well as the several advantages of observing parts with multiple levels and angulation and nondestructive virtual anatomy. It was possible to verify that the visualization of anatomical parts in more than one dimension helps to materialize abstract concepts, expanding the possibilities of understanding complex concepts of anatomy.
\end{abstract}

Keywords: Teaching anatomy; Pedagogical tools; Distance learning.

\title{
Resumen
}

La humanidad está atravesando un movimiento tecnológico nunca antes visto, en el que la información y la comunicación alcanzan un nivel esencial en la vida de las personas. Por lo tanto, existe la necesidad de que las escuelas se mantengan al día con los desarrollos tecnológicos y aprovechen al máximo los beneficios que es capaz de proporcionar. En este contexto, el presente trabajo tiene como tema, el uso de las Tecnologías de la Información y la Comunicación (TIC) en el ambiente de enseñanza-aprendizaje de la disciplina de Anatomía, más específicamente el uso de la simulación por computadora en las clases a distancia en los cursos de Salud. estudio de revisión a través de la plataforma científica Scielo, utilizando las siguientes palabras claves: "Simulación por Computador", "Enseñanza a Distancia" y "Enseñanza de Anatomía”. Se comprobó el papel de la simulación por ordenador en el estudio del cuerpo humano, en el que contribuye a aumentar la calidad de los resultados obtenidos y la reducción de costes y dificultades que conlleva, así como las diversas ventajas de observar partes con múltiples niveles. y angulación y anatomía virtual no destructiva. Se pudo comprobar que en lo que respecta al proceso de aprendizaje del alumno, la visualización de piezas anatómicas en más de una dimensión ayuda a materializar conceptos abstractos, ampliando las posibilidades de comprensión de conceptos complejos de Anatomía.

Palabras clave Enseñanza de la anatomía; Herramientas pedagógicas; Educación a distancia.

\section{Introduçãa}

A humanidade passa por uma movimentação tecnológica nunca vista até o momento, em que a informação e a comunicação alcançam um plano essencial na vida das pessoas. Atualmente, é difícil imaginar uma sociedade sem acesso à Internet. Sites de busca, redes sociais e e-mails fazem parte do nosso dia a dia e oferecem oportunidades de interação global.

Não se pode negar que ao longo dos anos a internet se popularizou e atingiu as casas de milhares de brasileiros em todas as camadas da sociedade. Facilitar a comunicação e divulgação de informações nas redes sociais. É importante aprofundar como os professores contextualizam sua prática pedagógica com as novas tecnologias (Cazeloto, 2019).

O avanço da tecnologia nas últimas décadas tem sido essencial para a sociedade, tornando possível maior agilidade na comunicação, otimização dos processos de produção, modernização de equipamentos, bem como facilitando execução de procedimentos médicos e pesquisas nos mais variados campos de conhecimento (Oliveira Cardoso \& Dickman, 2012).

Os séculos XX e XXI foram marcados por extraordinários e relevantes descobertas científicas. O desenvolvimento das ciências vem sendo marcado por importantes descobertas e avanços tecnológicos que têm contribuído para transformações significativas nas relações entre tecnologia e sociedade (Anjos, 2008). Destaca-se a necessidade das escolas em acompanhar a evolução tecnológica e tirar o máximo de proveito dos benefícios que esta é capaz de proporcionar (Oliveira Júnior et al., 2011). O século XXI proporcionou um momento de imersão tecnológica digital nunca antes imaginado, e a aprendizagem intercedida pela internet já está incorporada nos processos de ensino, no qual os recursos mais usados aproveitam as tecnologias digitais, através dos computadores conectados à internet

Sendo assim, as instituições de ensino públicas e privadas buscam constantemente se adequar as novas realidades ao incorporar recursos tecnológicos que permitam o ensino e a aprendizagem através de metodologias contextualizadas com a 
sociedade moderna, empregando, tal como, a internet e o computador como ferramentas de ensino para buscar um maior rendimento dos discentes.

Para Farias (2013), a Educação a Distância (EAD) é um sistema de aprendizagem com ênfase no aluno, permitindo que o discente tenha acesso às fontes de ensino, através de dispositivos eletrônicos e multimídia, fornecendo uma independência em termos de tempo e/ou espaço, bem como maior interação entre os alunos com o professor e/ou tutor, utilizando dispositivos eletrônicos. As Tecnologias de Informação e Comunicação (TIC) beneficiam o aumento da interatividade através do uso de ferramentas como imagens, textos, hipertextos, questionários, vídeos, áudios e jogos, em que juntam as tecnologias da informática e da telecomunicação (Lopes et al., 2013).

As Tecnologias de Informação e Comunicação representam os avanços tecnológicos e são empregadas em diversas áreas profissionais, assumindo assim, diversos significados e podem ser representadas por diversos métodos e ferramentas atuais. É notório que elas objetivam por meio do seu emprego a solução de problemas. Entretanto, entende-se que sua finalidade fundante por meio da aplicação do conhecimento científico que a originou é facilitar a convivência ou relações sociais da contemporaneidade.

Nesse contexto, destaca-se a utilização desses recursos tecnológicos nos cursos de saúde, mais especificamente, na disciplina de anatomia. O estudo da anatomia é uma das fundamentais na formação de profissionais da saúde, sendo um dos principais componentes curriculares para cursos da área da saúde, visto que esse conhecimento proporciona ao aluno domínio sobre detalhes estruturais para cada sistema de um determinado organismo (Antoniassi et al., 2020).

Logo, para a excelência em educação, faz-se necessária a interdisciplinaridade e a relação aluno-professor, tendo em vista a conexão em sala de aula. Para superar barreiras, as Instituições de Ensino Superior (IES), como estratégia de ensino, utilizam laboratórios práticos digitais, para que seus discentes pudessem intensificar seus estudos em diferentes horários e realizar monitorias de estudo (Antoniassi et al., 2020).

Nesse sentido, pretende-se com esse estudo analisar os benefícios da utilização de simuladores tecnológicos em substituição aos cadáveres nas aulas prática de Anatomia Humana através de simulação computacional, no ensino e aprendizagem a distância.

\section{Referencial teórico}

\subsection{Tecnologias de Informação e Comunicação (TIC) na Educação}

De acordo com Silva Dias (2020), o crescimento da utilização das Tecnologias de Informação e Comunicação (TICs) na educação é sequente de avanços tecnológicos que concederam o desenvolvimento de metodologias, de equipamentos e programas de computador que corroboraram com o desenvolvimento da educação no Brasil. Para os autores, a tendência atualmente, é que todas as tecnologias sejam compatíveis e possam ser produzidas e acessadas por uma única mídia, a chamada hipermídia.

Silva (2018) afirma que as TICs podem ser abrangidas a todas as tecnologias que fazem parte dos processos informacionais e comunicativos da sociedade, ou seja, um conjunto de recursos tecnológicos que interagem entre si, estando presentes em todo o mundo, nos negócios empresariais, nas faculdades, no campo, nas cidades, nos transportes e em todos os seguimentos do círculo social.

As TICs têm se apresentado uma possibilidade para favorecer o processo ensino-aprendizagem, tendo em vista que são recursos que estimulam os sentidos, permitem o compartilhamento de informações, apontam novas formas de relacionamento, de comunicação, de construção de conhecimentos e abrem novas possibilidades pedagógicas (Souza, 2015). As TICs na Educação são conceituadas como um conjunto de recursos tecnológicos, empregado de forma integrada, com um 
objetivo de facilitar a comunicação, o repasse de informações e o alcance de um alvo comum, alocando a educação como uma das áreas que mais se beneficia com sua implementação (Beira \& Nakamoto, 2016).

As TICs necessitam ser adotadas na educação de forma planejada, com o objetivo de conduzir os alunos à autonomia crescente, mediante o incentivo destes à pesquisa e à elaboração de seus trabalhos, mas que, para isso, o professor também carece de formação e capacitação sobre as TICs e suas possibilidades de uso na educação (Xavier, Teixeira, \& Saveti, 2010). As TICs podem ser utilizadas pelo professor como um instrumento intensificador do processo de ensino e aprendizagem, em que, por meio de sua introdução em Instituições de Ensino, há a possibilidade de se apresentar diferentes linguagens, como a audiovisual que está presente nos vídeos (Silva, 2019).

A Organização das Nações Unidas para a Educação, a Ciência e a Cultura (UNESCO) acreditam que as TIC podem colaborar para o acesso universal à educação, a equidade na educação, a qualidade de ensino e aprendizagem, o desenvolvimento profissional de professores, além de melhorar a gestão, a governança e a gestão educacional ao fornecer a combinação de políticas, tecnologias e capacidades (UNESCO, 2021).

\subsection{Ensino a Distância (EAD)}

A Educação a Distância (EAD) surgiu da necessidade de os alunos estudarem sem, necessariamente, estarem em uma sala de aula convencional, em determinado horário. Muitos não conseguiam desenvolver seus estudos no ensino fundamental, médio, educação de jovens e adultos (EJA) e nível superior devido às dificuldades de transporte, localização, assiduidade e frequência. Isso não quer dizer que o aluno não precise seguir critérios de organização, até porque existem cronogramas e uma série de atividades tais como: fóruns, trabalhos, áudios, leituras e vídeos a serem executados.

Litwin (2001, p. 13) define o Ensino a Distância como modalidade de ensino com propriedades que "substitui a proposta de assistência regular à aula por uma nova proposta, na qual os docentes ensinam e os alunos aprendem mediante situações não convencionais, ou seja, em espaços e tempos que não compartilham”. Para Behar (2009, p. 16), por sua vez, a EAD é uma forma de promover a "aprendizagem organizada, que se caracteriza, basicamente, pela separação física entre professor e alunos e a existência de algum tipo de tecnologia de mediação para estabelecer a interação entre eles". Essa modalidade de ensino inovadora tem a tecnologia como aliada contínua, selecionando, principalmente, qual ferramenta digital levará o estudante a ter uma aprendizagem significativa (Leal, 2020).

A Educação a Distância é considerada como uma forma democrática de permitir o acesso à educação a população que não é recebida pela escola tradicional, permitindo maior autonomia que pode ser oferecida aos alunos, de forma que consigam aperfeiçoar seus estudos, avançar nas pesquisas independentes e fazer descobertas sem a presença de um professor fisicamente, mas sim a distância (Andrade et al., 2020). Silva Coqueiro e Sousa (2021) elencam as principais características do EAD, que são:

- O Ensino a Distância emprega os recursos tecnológicos disponíveis, como exemplo as mídias, no qual podem ser integradas em ambientes conectados à internet, hospedados em plataformas de ensino;

- Estudantes e professores estão localizados em regiões distintas, encontrando-se presencialmente em datas previamente marcadas;

- As interações acontecem de forma virtual, podendo ser em tempo real (síncrona) ou em momentos distintos (assíncrona);

- A trilha estudantil do acadêmico é seguida pela equipe pedagógica, no qual composta por coordenadores, professores e tutores;

- O discente está no controle de seu processo de aprendizagem, desenvolvendo as habilidades para construir as competências elencadas nos planos dos cursos escolhidos; 


\section{Metodologia}

\subsection{Tipo de pesquisa}

Conforme caracteriza Köche (2016), do ponto de vista da natureza, esse trabalho trata de uma pesquisa básica, também conhecida como pesquisa fundamental, focada em ampliar o conhecimento que temos do mundo e tudo o que o forma. Esse tipo de pesquisa também é conhecido como fundamental, visto que objetiva acender conhecimentos para a ciência sem que estes tenham uma aplicação prática.

Pela perspectiva de abordagem, é uma pesquisa qualitativa, ou seja, é desenvolvido conceitos, ideias e entendimentos através de padrões encontrados nos dados, ao invés de coletar dados para comprovar teorias, hipóteses e modelos preconcebidos (Cardano, 2017). Analisando os objetivos essa pesquisa é exploratória, ou seja, a finalidade é proporcionar maior familiaridade com o problema, tornar-se explicito ou construir hipóteses com seu respeito ou causar aprimoramento do tema (Neto, 2017). Em relação aos procedimentos técnicos, é do tio Revisão Bibliográfica, no qual proporciona uma síntese de conhecimento e a incorporação dos resultados de estudos significativos, fundamentado em diversas pesquisas já publicadas.

\subsection{Procedimentos metodológicos}

Os procedimentos metodológicos de um trabalho acadêmico é um processo lógico com finalidade principal de atingir um determinado fim ou para se chegar ao conhecimento de um determinado assunto (Assunção et al., 2016). O método é uma forma de proceder ao longo de um caminho. Na ciência os métodos constituem os instrumentos básicos que ordenam o pensamento em sistemas, traçam de modo ordenado a maneira de proceder do cientista ao longo de um percurso para alcançar um objetivo.

Para a realização desse estudo, a primeira etapa foi a organização do problema a ser pesquisado, para posteriormente avaliar e aplicar todo o máximo do material bibliográfico disponível, uma vez que o tema deve conter relevância tanto teórica como prática e proporcionar interesse de ser estudado (Lemos, 2018). Nesse sentido, será utilizado trabalhos científicos acerca do tema, através de plataformas cientificas, como Scielo e o Google Acadêmico, no período indeterminado, tendo as seguintes palavras-chave: "Simulação Computacional", "Ensino a Distância" e "Ensino de Anatomia".

Para organizar as informações dos trabalhos selecionados da base dados, foi utilizada a leitura flutuante dos títulos e resumos dos trabalhos bem como os resultados apresentados. O principal critério de exclusão de artigos está relacionado ao tempo da publicação, visto que apenas obras mais recentes são bem vistas no meio cientifico. A ordem de prioridade para a escolha de trabalho foi: (i) artigos publicados em periódicos internacionais; (ii) artigos publicados em periódicos nacionais reconhecidos; (iii) livros publicados por bons editores; (iv) teses e dissertações; (v) anais de conferências internacionais; (vi) anais de conferências nacionais.

Critérios de inclusão: estudos encontrados na base de dado escolhida, publicados em período indeterminado, utilizando os descritores já citados. Critérios de exclusão: artigo noticiosos, textos em resenhas, artigos não indexados, opiniões, editoriais ou manuais. Para organizar as informações dos trabalhos selecionados da base dados, será utilizada a leitura flutuante dos títulos e resumos dos trabalhos, bem como os resultados apresentados.

No que se refere a delimitação temporal, o presente trabalho realizará uma pesquisa sobre o tema nos últimos 10 anos. Os resultados desta pesquisa geraram um conjunto inicial de documentos, que filtraremos os resultados iniciais da pesquisa a partir da leitura do título e do resumo. Todos os artigos encontrados, relacionados ao tema, foram incluídos na análise, independentemente de ser o assunto principal do artigo ou apenas mencionado no resumo.

\section{Resultados e discussões}


Fornaziero et al. (2010, p. 291), ao tratar sobre a constante evolução da tecnologia educacional em consonância com o ensino da Anatomia Humana, afirma que:

A constante evolução da tecnologia educacional faz emergir a necessidade de apreensão, clarificação e possíveis adaptações dos métodos de ensino-aprendizagem. Para tanto, acredita-se haver possibilidade de modificações das práticas pedagógicas, visto que as mudanças já estão inseridas no dia a dia dos estudantes e que o processo ensinoaprendizagem deve ser condizente com a realidade que o acadêmico vivencia em sua rotina. Este processo deve ser largamente discutido entre os professores para que se apliquem propostas pedagógicas coerentes, instigando a transformação do desempenho dos sujeitos em sala de aula.

A Simulação pode ser conceituada como a construção e manipulação operacional de um modelo, ou seja, uma representação física ou simbólica de aspectos de um processo físico e ou social (Júnior et al., 2012). "Os softwares podem ser utilizados devido a sua interatividade e capacidade de vivenciar situações, criando modelos, discutindo-os, testando-os, apresentando e interpretando suas possíveis soluções" (Cavalcante et al., 2017, p. 3).

Segundo Agra et al. (2019), as simulações computacionais estão associadas a Teoria da Aprendizagem Significativa, no qual trata-se de uma estratégia em situação formal de ensino, em que incide na interação não arbitrária e não literal de novos conhecimentos com conhecimentos prévios. Assim, a partir de sucessivas interações, um determinado conhecimento prévio, progressivamente, adquire novos significados, torna-se mais rico, mais refinado, mais diferenciado e é capaz de servir de âncora para novas aprendizagens significativas. Ou seja, dentre todos os fatores que influenciam a aprendizagem, o mais importante é o que o aluno previamente sabe; aspecto considerado principal e ponto de partida.

Por meio de simulações computacionais, é imaginável criar as condições necessárias para que a aprendizagem significativa ocorra, no qual as atividades de modelagem e simulação estão entre as formas mais criativas, benéficas e que geram interação de aplicações na Educação (Souza \& Dandolini, 2009). Para os autores, esses sistemas informatizados criam formas de representação que tornam possível o uso didático de certo conteúdo de difícil entendimento, sem necessidade de habilidades especiais em programação matemática.

Rodrigues et al. (2018) afirmam que as ferramentas de simulação computacional são um aspecto essencial da utilização da informática na educação, em que possui maior utilidade na demonstração de fenômenos, representação de situações mais complexas ou que apresentam maior perigo em seu manuseio, difíceis de serem realizadas em laboratórios através de experimentos reais. Os autores ainda complementam ao afirmar que a aprendizagem fundamentada em simulação envolve a aprendizagem realizada em um ambiente informatizado, em que o discente interage com as entidades do ambiente e, gradativamente, pode induzir nas características do modelo do conceito, enquanto ele prossegue através da simulação, o que pode levar a mudanças no seu conceito original.

\subsection{Simulação computacional aplicado no ensino e aprendizagem de Anotomia}

Durante o século XX, não ocorreram mudanças expressivas nos métodos de ensino de anatomia nas Instituições de Ensino Superior, com predomínio de palestras expositivas e atividades laboratoriais de anatomia. Com o advento de novas tecnologias, simuladores, realidade aumentada e realidade virtual, surgiram novas possibilidades de ensino que podem auxiliar e, em algumas situações, substituir os métodos tradicionais de ensino (Duarte et al., 2020). Nesse sentido, o ensino de anatomia está passando por mudanças significativas devido às limitações de tempo, disponibilidade limitada de cadáveres e desenvolvimentos tecnológicos nas áreas de modelagem tridimensional e aprendizagem assistida por computador.

De acordo com a história, o estudo da anatomia é efetivado com uso de cadáveres, entretanto, existem algumas desvantagens dessa prática, como o alto custo para manutenção das peças, a dificuldade no armazenamento de maneira adequada, quantidade insuficiente de peças em razão da dificuldade na sua aquisição e demanda crescente, a degradação 
causada pelo manuseio constante das peças e o uso de produtos químicos tóxicos para a conservação (Venâncio \& Bittencourt, 2020). A simulação computacional no estudo do corpo humano contribui com o aumento da qualidade dos resultados obtidos e a redução de custos e dificuldades envolvidos, em que a fonte de dados deve ser rica em informações e em imagens (Oliveira et al., 2009).

De acordo com Santos (2010), os primeiros sistemas de Realidade Virtual para ensino na saúde foram os atlas tridimensionais, em que apresentavam uma visualização inovadora de aspectos da anatomia e fisiologia humana. Posteriormente, com a evolução do potencial de processamento dos computadores pessoais e com o desenvolvimento de novos dispositivos de interação tridimensional, começaram a ser propostos os simuladores virtuais, em que se baseiam na imersão do usuário em um sistema de Realidade Virtual tridimensional e interativo, visando oferecer aprendizado real ao estudante.

Logo, surgiu nas últimas décadas a necessidade do ensino de anatomia por meio do uso de tecnologia, da realidade virtual (VR) para construir um sistema de ensino de anatomia virtual, baseado no conjunto de dados chinês "Chinese Visible Human" (CVH), no qual pode fornecer recursos de ensino reais e reutilizáveis para o ensino da anatomia. Esse ensino virtual de Anatomia possui diversas vantagens de observação de peças com múltiplos níveis e angulação e anatomia virtual não destrutiva (Venâncio \& Bittencourt, 2020).

Destaca-se, dentre essas ferramentas tecnológicas aplicadas no ensino de saúde, os modelos de visualização 3D, no qual é preciso fornecer conectividade entre as fontes dados e usuários finais, seja através de aplicações concretas ou através de interface web. Nesses modelos, é possível explorar e realizar simulações de acordo com as opções e atributos e para visualizar o impacto do concreto problema nessas ferramentas (Brazina et al., 2014).

Em um estudo com objetivo de construir um Laboratório de Anatomia em 3D, contextualizado com material didáticopedagógico para ser utilizado como apoio na EaD através de aplicações educacionais em 3D com o uso do Metaverso Second Life, Massaro et al. (2011) verificaram que as experiências já realizadas com o Second Life nos ambientes educacionais demonstram o potencial de interação e imersão, visto que ao possibilitar diversas formas de comunicação, apresenta-se como uma alternativa para minimizar a falta de presença física do professor. O Second Life admite uma sensação de presença, desperta o sentimento de estar em relação com o outro, aumentando o grau de envolvimento dos sujeitos envolvidos no ambiente, que pode contribuir para potencializar a experiência de aprendizagem da Anatomia.

Além disso, Massaro et al. (2011) constataram que a construção de aplicações educacionais em 3D com conteúdo interativos, por meio de simulações, corroboram para otimizar os processos de ensino e aprendizagem relacionados aos conceitos da área de estudo do corpo humano. No que se refere o processo de aprendizagem do discente, os autores verificaram que a visualização das peças anatômicas em mais de uma dimensão auxilia a materializar conceitos abstratos, ampliando as possibilidades de compreensão de conceitos complexos de Anatomia.

Fornaziero e Gil (2021), em um estudo com finalidade de verificar as inovações tecnológicas que podem ser utilizadas para tornar o aprendizado de Anatomia Humana, empregaram simulações para o ensino de Anatomia Humana na formação de profissionais de saúde. Os autores verificaram existem algumas justificativas que colaboram para o uso de simulações:

- $\quad$ O uso de cadáveres reais causa pesadelos, insônia e depressão nos estudantes;

- $\quad$ Estudantes relataram que os cadáveres provocam ansiedade e repulsa visual;

- O procedimento complexo para se adquirir um cadáver, visto que envolve, além de aspectos legais, a questão religiosa.

Além disso, Fornaziero e Gil (2021) identificaram que a junção de programas de computador com os trabalhos práticos contribui de forma expressiva no conhecimento teórico dos ternas morfológicos, bem como as simulações computacionais orientam e facilitam a compreensão dos conteúdos. Nesse contexto, a percepção dos alunos é de que a 
docência atualizada e eficiente não coloca de lado os métodos tradicionais da prática docente, ou seja, eles continuam a dizer que consideram relevante o uso de computadores, mas acham essencial a prática de anatomia.

\section{Considerações Finais}

O presente estudo buscou, a partir de um estudo exploratório e de caráter bibliográfico, analisar os benefícios da utilização de simuladores tecnológicos em substituição aos cadáveres nas aulas prática de Anatomia Humana através de simulação computacional, no ensino e aprendizagem a distância.

A partir desse estudo bibliográfico, verificou-se que as Tecnologias de Informação e Comunicação (TIC) colaboram positivamente com o aumento da interatividade através do uso de ferramentas como imagens, textos, hipertextos, questionários, vídeos, áudios e jogos, ferramentas essas essenciais na Educação a Distância.

No que se refere a utilização de ferramentas computacionais no ensino e aprendizagem de Anatomia, percebeu-se grande atenção de pesquisadores nesse tema, no qual destaca-se o papel da simulação computacional no estudo do corpo humano, contribuindo com o aumento da qualidade dos resultados obtidos e a redução de custos e dificuldades envolvidos, bem como as diversas vantagens de observação de peças com múltiplos níveis e angulação e anatomia virtual não destrutiva.

Logo, foi possível verificar que no que se refere o processo de aprendizagem do discente, a visualização das peças anatômicas em mais de uma dimensão auxilia a materializar conceitos abstratos, ampliando as possibilidades de compreensão de conceitos complexos de Anatomia.

\section{Referências}

Agra, G. et al. (2019). Análise do conceito de Aprendizagem Significativa à luz da Teoria de Ausubel. Revista Brasileira de Enfermagem, 72, $248-255$.

Andrade, S. et al. (2020). Os desafios do Ensino à Distância e do uso da Tecnologia de Informação e Comunicação. Revista de Casos e Consultoria, 11(1), e11119-e11119.

Anjos, A. J. S. (2008). As novas tecnologias e o uso dos recursos telemáticos na educação científica: a simulação computacional na educação em Física. Caderno Brasileiro de Ensino de Física, 25(3), 569-600.

Antoniassi, E. S. et al. (2021). Perspectivas de alunos e professores da área da saúde sobre ensino e aprendizado de práticas anatômicas à distância. Brazilian Journal of Animal and Environmental Research, 4(1), 1483-1499.

Assunção, G. H., Reis, M. A. A., \& De Abreu, M. C. S. (2016). Disgrafia, Discalculia E Dislexia: Suas Implicações na Educação Infantil. Linha de Pesquisa: $5^{a}$-Estudos Culturais e Linguagens na Educação, p. 748.

Behar, P. A. (2009). Modelos pedagógicos em educação a distância. Artmed Editora.

Beira, D., \& Nakamoto, Paula. (2016). A Formação docente inicial e continuada prepara os Professores para o Uso das Tecnologias de Informação e Comunicação (TICs) em sala de aula? In: Anais do Workshop de Informática na Escola, p. 825.

Brazina, D., Fojtik, R., \& Rombova, Z. (2014). Visualização 3D no ensino de anatomia. Procedia-Social and Behavioral Sciences, 143, 367-371.

Cardano, M. (2017). Manual de pesquisa qualitativa. A contribuição da teoria da argumentação. Tradução: Elisabeth da Rosa Conill. Vozes.

Cavalcante, F. F., Andrade, L. S., \& Geller, M. A utilização da simulação computacional como estratégia de ensino nas aulas de física. https://www.researchgate.net/profile/Marlise-Geller/publication/228417908_A_UTILIZACAO_DA_SIMULACAO_COMPUTACIONAL_COMO_EST RATEGIA_DE_ENSINO_NAS_AULAS_DE_FISICA/links/55da43e408aeb38e8a8a116b/A-UTILIZACAO-DA-SIMULACAO-COMPUTACIONALCOMO-ESTRATEGIA-DE-ENSINO-NAS-AULAS-DE-FISICA.pdf

Cazeloto, E. (2019). Inclusão digital: uma visão crítica. Editora Senac São Paulo.

Duarte, M. L. et al. (2020). Learning anatomy by virtual reality and augmented reality. A scope review. Morphologie.

Farias, S. C. (2013). Os benefícios das tecnologias de informação e comunicação (TIC) no processo de educação a distância (EAD). RDBCI: Revista Digital de Biblioteconomia e Ciência da Informação, 11(3), 15-29.

Fornaziero, C. C. et al. (2010). O ensino da anatomia: integração do corpo humano e meio ambiente. Revista Brasileira de Educação Médica, $34,290-297$.

Fornaziero, C. C., \& Gil, C. R. R. (2021). Novas tecnologias aplicadas ao ensino da anatomia humana. Revista Brasileira de Educação Médica, $27,141-146$. 
Júnior, N. T., De Souza, G. G., \& Do Nascimento, J. Z. (2012). Análise de processos por meio da simulação computacional: proposta de uma estratégia de ensino na graduação em Administração. Administração: Ensino e Pesquisa, 13(3), 491-521.

Köche, J. C. (2016). Fundamentos de metodologia científica. Editora Vozes.

Leal, P. S. (2020). A Educação Diante De Um Novo Paradigma: Ensino A Distância (EAD) Veio Para Ficar! Revista Gestão \& Tecnologia, 1(30), 41-43.

Lemos, F. D. (2018). A questão epistemológica do pesquisador que pesquisa dentro da sua organização. Anais do Interprogramas Secomunica, 2.

Llitwin, E. (2001). Educação a Distância: Temas para debate de uma nova agenda educativa. Artmed.

Lopes, R. T., Pereira, A. C., \& Silva, M. A. D. da. (2013). O uso das TIC no ensino da morfologia nos cursos de saúde do Rio Grande do Norte. Revista Brasileira de Educação Médica, 37(3), 359-364.

Massaro, G., Mantovani, A. M., \& Da Silva Rodrigues, Marcello. (2011). Aplicações educacionais em 3D para os processos de ensino e aprendizagem da área de anatomia no second life. RENOTE, 9(2).

Neto, J. A. M. (2017). Metodologia científica na era da informática. Saraiva Educação SA.

Oliveira Cardoso, S. O., \& Dickman, A. G. (2012). Simulação computacional aliada à teoria da aprendizagem significativa: uma ferramenta para ensino e aprendizagem do efeito fotoelétrico. Caderno Brasileiro de Ensino de Física, 891-934.

Oliveira Júnior, F. M. et al. (2011). O Uso de Simulações Computacionais Como Ferramenta de Ensino e Aprendizagem dos Conceitos de Circuitos Elétricos. XIX Simpósio Nacional de Ensino de Física-SNEF, 19(1).

Oliveira, I. K., De Oliveira, H. J. Q., \& Luque, L. Modelos de sistemas anatômicos para simulação computacional voltados ao treinamento de estudantes: submódulo gestão de aulas. https://www.umc.br/_img/_diversos/pesquisa/pibic_pvic/XII_congresso/projetos/Itamar_Kadota.pdf

Rodrigues, J. J. V. et al. (2018). Simulações computacionais e mapas conceituais no auxílio à aprendizagem significativa do conceito de energia. Experiências em Ensino de Ciências, 13(5), 535-554.

Santos, A. D. dos. (2010). Simulação Médica Baseada em Realidade Virtual para Ensino e Treinamento em Ginecologia. 2010.154 f. Dissertação (Mestrado em Informática) - Universidade Federal da Paraí-ba, João Pessoa.

Silva Coqueiro, N. P., \& Sousa, E. C. (2021). A educação a distância (EAD) e o ensino remoto emergencial (ERE) em tempos de Pandemia da Covid 19. Brazilian Journal of Development, 7(7), 66061-66075.

Silva Dias, V. et al. (2020). Tecnologias da Informação e Comunicação (TICs) e a inovação das políticas públicas educacionais. Brazilian Journal of Development, 6(11),. 90819-90837.

Silva, C. G. da. (2018). A Importância do Uso das TICS Na Educação. Revista Científica Multidisciplinar Núcleo do Conhecimento, 3(8), 49-59.

Silva, L. E. L. (2019). A concepção dos discentes do curso de Licenciatura em Ciências - Biologia e Química do Instituto de Educação, Agricultura e Ambiente (IEAA-UFAM) sobre o uso das tecnologias de informação e comunicação (TICs) no ensino de química. $37 \mathrm{f}$. Trabalho de Conclusão de Curso Universidade Federal do Amazonas.

Souza, A. M. (2015). As Tecnologias da Informação e da Comunicação (TIC) na educação para todos. Educação em Foco, 349-366.

Souza, J. A., \& Dandolini, G. A. (2009). Utilizando simulação computacional como estratégia de ensino: estudo de caso. RENOTE, 7(1).

UNESCO. TIC na educação do Brasil. https://pt.unesco.org/fieldoffice/brasilia/expertise/ict-education-brazil

Venâncio, D. C. M. (2020). O uso de simuladores tecnológicos em disciplinas de anatomia. https://repositorio.ifsc.edu.br/bitstream/handle/123456789/2153/Denise\%20Corr\%C3\%AAa\%20Martins\%20Ven\%C3\%A2ncio.pdf?sequence=1\&isAllowed= $\mathrm{y}$

Xavier, M. C., Teixeira, C. R., \& Saveti, B. P. (2010). Aplicação das Tecnologias da Informação e Comunicação (TICs) na educação e os desafios do educador. Dialogia, 9(1), 105-116. 